\title{
SPECTRA OF OPERATORS WITH FIXED IMAGINARY PARTS
}

\author{
ANDRZEJ POKRZYWA
}

\begin{abstract}
The aim of this paper is to obtain the best bound for the distance between the eigenvalues of a Hermitian matrix $B$ and the real parts of eigenvalues of a matrix $B+i A$, where $A$ is also Hermitian, in the terms of eigenvalues of $A$. A similar problem in infinite-dimensional Hilbert space is also considered.
\end{abstract}

This paper was inspired by the papers of Kahan [4], [5] and Gohberg [1]. The obtained results may be regarded as the generalizations of some results of these authors. A solution of the problem of Kahan, which consists of computing the best constant in the inequality $\left\|Z-Z^{*}\right\|<K_{n}\left\|Z+Z^{*}\right\|$ for all $n \times n$ matrices with real spectrum, is obtained (Corollary 2).

Notations. Let $H$ denote a complex Hilbert space with the norm $\|\cdot\|$ and the scalar product $\langle\cdot, \cdot\rangle \cdot L(H)$ denotes the algebra of all bounded linear operators acting in $H$. For an $A \in L(H), \sigma(A)$ denotes the spectrum of $A$. For a compact $A \in L(H), s_{1}, s_{2}, \ldots$ denote the eigenvalues of $\sqrt{A A^{*}}$, repeated according to multiplicity and arranged in decreasing order.

Finite-dimensional case. In this section we assume that $\boldsymbol{H}$ is $\boldsymbol{n}$-dimensional and that $A=A^{*}$ is an operator in $H$ with eigenvalues $\lambda_{1}>\lambda_{2}>\cdots>\lambda_{n}$.

LEMMA 1. There exists $B=B^{*} \in L(H)$ such that $\sigma(A+i B) \subset \mathbf{R}$ and

$$
\|B\|=\frac{1}{n} \sum_{j=1}^{n} \lambda_{j} \operatorname{ctg} \frac{2 j-1}{2 n} \pi .
$$

Proof. Let $\left\{e_{j}\right\}_{1}^{n}$ be an orthonormal basis of $H$. Define operator $S$ by the formula

$$
S=-\left\langle\cdot, e_{1}\right\rangle e_{n}+\sum_{2}^{n}\left\langle\cdot, e_{j}\right\rangle e_{j-1}
$$

The vectors $v_{k}=\sum_{j=1}^{n} \exp (((2 k-1) / n) j \pi i) e_{j}$ are mutually orthogonal eigenvectors of $S$ of norm $n$. When the basis $\left\{e_{j}\right\}$ is suitably chosen then

$$
A=\frac{1}{n} \sum_{1}^{n} \lambda_{j}\left\langle\cdot, v_{j}\right\rangle v_{j}
$$

Received by the editors May 25, 1979.

AMS (MOS) subject classifications (1970). Primary 15A42, 15A60, 47A55; Secondary 15A18, 15 A57. 
We shall show that the operator

$$
B=i \sum_{k, j=1}^{n} \operatorname{sign}(k-j)\left\langle A e_{j}, e_{k}\right\rangle\left\langle\cdot, e_{j}\right\rangle e_{k}
$$

satisfies the thesis of our lemma.

Since $A=\sum_{k_{j}=1}^{n}\left\langle A e_{j}, e_{k}\right\rangle\left\langle\cdot, e_{j}\right\rangle e_{k}$ we see that the matrix of the operator

$$
A+i B=\sum_{k=1}^{n}\left\langle A e_{k}, e_{k}\right\rangle\left\langle\cdot, e_{k}\right\rangle e_{k}+2 \sum_{k<j}\left\langle A e_{j}, e_{k}\right\rangle\left\langle\cdot, e_{j}\right\rangle e_{k}
$$

is triangular in the basis $\left\{e_{j}\right\}$. Therefore the eigenvalues of $A+i B$ are $\lambda_{k}(A+i B)$ $=\left\langle A e_{k}, e_{k}\right\rangle=(1 / n) \Sigma_{1}^{n} \lambda_{j}$. Hence $\sigma(A+i B) \subset \mathbf{R}$.

Note that

$$
\left\langle A e_{j}, e_{k}\right\rangle=\left\langle\frac{1}{n} \sum_{s=1}^{n} \lambda_{s}\left\langle e_{j}, v_{s}\right\rangle v_{s}, e_{k}\right\rangle=\frac{1}{n} \sum_{s=1}^{n} \lambda_{s} \exp \left(\frac{2 s-1}{n}(k-j) \pi i\right) .
$$

Now it is easy to verify that if we define the numbers $b_{j}$ and the operator $T$ by the relations

$$
b_{j}=-i \sum_{s=1}^{n} \lambda_{s} \exp \left(-\frac{2 s-1}{n} j \pi i\right), \quad T=\left\langle\cdot, e_{1}\right\rangle e_{n}+\sum_{k=1}^{n-1}\left\langle\cdot, e_{k+1}\right\rangle e_{k},
$$

then $B=(1 / n) \sum_{j=1}^{n-1} b_{j} T^{j}$. Since the numbers $\lambda_{k}(T)=\exp (2 k \pi i / n)$ are the eigenvalues of $T$, the eigenvalues of $B$ are

$$
\lambda_{k}(B)=\frac{1}{n} \sum_{j=1}^{n-1} b_{j}\left(\lambda_{k}(T)\right)^{j}=\frac{1}{n} \sum_{s=1}^{n} \lambda_{s} \operatorname{ctg} \frac{2(s-k)-1}{2 n} \pi .
$$

Hence

$$
\|B\|=\max _{k}\left|\lambda_{k}(B)\right|=\frac{1}{n} \sum_{s=1}^{n} \lambda_{s} \operatorname{ctg} \frac{2 s-1}{2 n} \pi .
$$

Lemma 2. If $B=B^{*} \in L(H)$ and $\sigma(A+i B) \subset \mathbf{R}$ then

$$
\|B\| \leqslant \frac{1}{n} \sum_{s=1}^{n} \lambda_{s} \operatorname{ctg} \frac{2 s-1}{2 n} \pi .
$$

Proof. By the theorem on triangular matrix form there exists an orthonormal basis $\left\{e_{j}\right\}_{1}^{n}$ of $H$ such that $\left\langle(A+i B) e_{k}, e_{j}\right\rangle=0=\left\langle(A-i B) e_{j}, e_{k}\right\rangle$ for $k<j$. This implies that $i\left\langle B e_{j}, e_{k}\right\rangle=\left\langle A e_{j}, e_{k}\right\rangle$ and $i\left\langle B e_{k}, e_{j}\right\rangle=-\left\langle A e_{k}, e_{j}\right\rangle$ for $k\langle j$. Since $\left\langle A+i B e_{k}, e_{k}\right\rangle \subset \sigma(A+i B) \subset \mathbf{R}$ and $A, B$ are selfadjoint, $\left\langle B e_{k}, e_{k}\right\rangle=0$. Hence $B=\Sigma_{j, k}\left\langle B e_{j}, e_{k}\right\rangle\left\langle\cdot, e_{j}\right\rangle e_{k}=-\Sigma_{j, k} \operatorname{sign}(j-k)\left\langle A e_{j}, e_{k}\right\rangle\left\langle\cdot, e_{j}\right\rangle e_{k}$. Setting $\left\langle\cdot, e_{j}\right\rangle e_{j}=E_{j}$ we may write

$$
B=i \sum_{j, k} \operatorname{sign}(k-j) E_{k} A E_{j}
$$

Since $B=B^{*}$ there is a unit eigenvector $f$ of $B$ such that $\|B\|=|\langle B f, f\rangle|$. If we set $\langle\cdot, f\rangle f=F$ then $\operatorname{tr} B F=\langle B f, f\rangle$. Using properties of trace and (1) we see 
that

$$
\begin{aligned}
( \pm\|B\|=) \operatorname{tr} B F & =\operatorname{tr}\left(i \sum_{j, k} \operatorname{sign}(k-j) E_{k} A E_{j} F\right) \\
& =\operatorname{tr}\left(A \sum_{j, k} i \operatorname{sign}(k-j) E_{j} F E_{k}\right)=\operatorname{tr} A G
\end{aligned}
$$

where we have set $i \sum_{j, k} \operatorname{sign}(k-j) E_{j} F E_{k}=G$. $G$ is a selfadjoint operator. Let $\omega_{1} \geqslant \omega_{2} \geqslant \cdots \geqslant \omega_{n}$ be its eigenvalues. It is shown in [1] that

$$
\omega_{j}=-\omega_{n+1-j}, \quad j=1,2, \ldots, n,
$$

and that if $\left\|E_{j} f\right\| \neq 0$ for all $j$ then for $j<[n / 2] \sum_{k=1}^{n} \arg \left(\omega_{j}+i\left\|E_{k} f\right\|^{2}\right)=$ $(2 j-1) \pi / 2$. This means that

$$
\begin{aligned}
\frac{(2 j-1) \pi}{(2 n)} & =\frac{1}{n} \sum_{k=1}^{n} \operatorname{arctg}\left(\left\|E_{k} f\right\|^{2} / \omega_{j}\right) \\
& <\operatorname{arctg}\left(\frac{1}{n} \sum_{k=1}^{n}\left\|E_{k} f\right\|^{2} / \omega_{j}\right)=\operatorname{arctg} \frac{1}{n \omega_{j}},
\end{aligned}
$$

since the function arc tg is concave in the interval $[0, \infty]$. Since tangent is an increasing function in $[0, \pi / 2)$ we obtain the inequality $\operatorname{tg}((2 j-1) \pi /(2 n))<$ $1 /\left(n \omega_{j}\right)$, equivalent to

$$
\omega_{j} \leqslant \frac{1}{n} \operatorname{ctg} \frac{2 j-1}{2 n} \pi, \quad j=1,2, \ldots, n / 2 .
$$

By continuity of eigenvalues (4) holds also when $E_{j} f=0$ for some $j$.

It follows from (3) that $\operatorname{tr} G=0$. Let $\mu>-\lambda_{n}$; then

$$
s_{j}(A+\mu)=\lambda_{j}+\mu \text {. }
$$

Let $x_{j}$ be the normalized eigenvector of $G, G x_{j}=\omega_{j} x_{j}$. Since $G$ is selfadjoint $\left\{x_{j}\right\}_{1}^{n}$ is an orthonormal basis for $H$. Thus, using Abel transformation, we may write

$$
\begin{aligned}
\operatorname{tr} A G & =\operatorname{tr}(A+\mu) G=\sum_{j}\left\langle(A+\mu) G x_{j}, x_{j}\right\rangle=\sum_{j} \omega_{j}\left\langle(A+\mu) x_{j}, x_{j}\right\rangle \\
& =\sum_{j=1}^{n-1}\left(\omega_{j}-\omega_{j+1}\right) \sum_{k=1}^{j}\left\langle(A+\mu) x_{k}, x_{k}\right\rangle+\omega_{n} \sum_{k=1}^{n}\left\langle(A+\mu) x_{k}, x_{k}\right\rangle .
\end{aligned}
$$

The Ky-Fan lemma [2, Lemma II.4.1] and (5) imply that

$$
\left|\sum_{k=1}^{j}\left\langle(A+\mu) x_{k}, x_{k}\right\rangle\right|<\sum_{k=1}^{j} s_{k}(A+\mu)=\sum_{k=1}^{j}\left(\lambda_{k}+\mu\right) .
$$

Note also that if $j=n$ then in the above inequality we have in fact the equality without the modulus. Hence

$$
\begin{aligned}
\operatorname{tr} A G & \leqslant \sum_{j=1}^{n-1}\left(\omega_{j}-\omega_{j+1}\right) \sum_{k=1}^{j}\left(\lambda_{k}+\mu\right)+\omega_{n} \sum_{k=1}^{n}\left(\lambda_{k}+\mu\right) \\
& =\sum_{j=1}^{n} \omega_{j}\left(\lambda_{j}+\mu\right)=\sum_{j=1}^{n} \lambda_{j} \omega_{j} .
\end{aligned}
$$


Writing the just obtained inequality with $-A$ instead of $A$ we obtain by (3) the inequality $-\operatorname{tr} A G \leqslant \sum_{j} \omega_{j}\left(-\lambda_{n+1-j}\right)=\sum_{j} \lambda_{j} \omega_{j}$. This with (2), (3) and (4) shows that

$$
\begin{aligned}
\|B\| & =|\operatorname{tr} A G| \leqslant \sum_{j} \lambda_{j} \omega_{j}=\sum_{j=1}^{[n / 2]}\left(\lambda_{j}-\lambda_{n+1-j}\right) \omega_{j} \\
& \leqslant \frac{1}{n} \sum_{1}^{[n / 2]}\left(\lambda_{j}-\lambda_{n+1-j}\right) \operatorname{ctg} \frac{2 j-1}{2 n} \pi=\frac{1}{n} \sum_{1}^{n} \lambda_{j} \operatorname{ctg} \frac{2 j-1}{2 n} \pi .
\end{aligned}
$$

The lemma is proved.

THEOREM 1. Suppose that $B$ is a selfadjoint operator in $H$. Let $\left\{\beta_{j}\right\}_{1}^{n},\left\{\mu_{j}\right\}_{1}^{n}$ be the eigenvalues of $B, B+i A$, respectively, arranged in such a way that $\beta_{j} \geqslant \beta_{j+1}$, $\operatorname{Re} \mu_{j} \geqslant \operatorname{Re} \mu_{j+1}$ for $j=1,2, \ldots, n-1$. Then

$$
\left|\beta_{j}-\operatorname{Re} \mu_{j}\right|<\frac{1}{n} \sum_{s=1}^{n} \lambda_{s} \operatorname{ctg} \frac{2 s-1}{2 n} \pi .
$$

Proof. Following Kahan [5] and identifying the operators with matrices we may assume that $B+i A$ is an upper triangular matrix and that $B+i A=D+i Z$, where $D$ is a real diagonal matrix, $Z$ is an upper triangular matrix with real spectrum. Hence the numbers $\operatorname{Re} \mu_{j}$ are eigenvalues of $D$. Since $B-D=$ $i\left(Z-Z^{*}\right) / 2$ it follows from Weyl's inequality that $\left|\beta_{j}-\operatorname{Re} \mu_{j}\right|<\|B-D\|=$ $\|\operatorname{Im} Z\|$. Since $\operatorname{Re} Z=\left(Z+Z^{*}\right) / 2=A$ the thesis follows from Lemma 2 .

For a subset $F$ of the complex plane let $\operatorname{Re} F=\{\operatorname{Re} \lambda ; \lambda \in F\}$. The following corollaries follow easily from the obtained results.

Corollary 1.

$$
\max _{B=B^{*} \in L(H)} \operatorname{dist}(\sigma(B), \operatorname{Re} \sigma(B+i A))=\frac{1}{n} \sum_{1}^{n} \lambda_{s} \operatorname{ctg} \frac{2 s-1}{2 n} \pi .
$$

(dist $(\cdot, \cdot)$ denotes the Hausdorff distance of sets.)

Corollary 2.

$$
\begin{aligned}
K_{n} & =\max \left\{\left\|Z-Z^{*}\right\| /\left\|Z+Z^{*}\right\| ; Z \in L(H), \sigma(Z) \subset \mathbf{R}, Z \neq 0\right\} \\
& =\frac{2}{n} \sum_{1}^{[n / 2]} \operatorname{ctg} \frac{2 s-1}{2 n} \pi ; \\
K_{n} & =\max \left\{\operatorname{dist} \sigma(B), \operatorname{Re} \sigma(B+i C) ; B=B^{*}, C=C^{*},\|C\|<1\right\} .
\end{aligned}
$$

Using the inequalities

$$
\begin{aligned}
\int_{(2 s-1) \pi /(2 n)}^{(2 s+1) \pi /(2 n)} \operatorname{ctg} x & \leqslant \frac{\pi}{2 n}\left(\operatorname{ctg} \frac{2 s+1}{2 n} \pi+\operatorname{ctg} \frac{2 s-1}{2 n} \pi\right), \\
\frac{\pi}{n} \operatorname{ctg} \frac{2 s-1}{2 n} & \leqslant \int_{(s-1) \pi / n}^{(s+1) \pi / n} \operatorname{ctg} x \quad(1<s<[n / 2]),
\end{aligned}
$$

one may see that

$$
\frac{1}{n} \operatorname{ctg} \frac{\pi}{2 n}-\frac{2}{\pi} \ln \sin \frac{\pi}{2 n}<K_{n}<\frac{2}{n} \operatorname{ctg} \frac{\pi}{2 n}-\frac{2}{\pi} \ln \sin \frac{\pi}{n}
$$

and that $K_{n} / \ln n \rightarrow 2 / \pi$. 
Infinite-dimensional case. Let $H$ be a separable infinite-dimensional Hilbert space, and let $A$ be a selfadjoint compact operator in $H . \lambda_{j}^{+}, \lambda_{j}^{-}, j=$ $1,2, \ldots$, denote the positive eigenvalues of $A$ and $-A$, respectively, repeated according to multiplicity and arranged in decreasing order. If there are only $n$ positive (negative) eigenvalues of $A$ we set $\lambda_{j}^{+}=0\left(\lambda_{j}^{-}=0\right)$ for $j>n$.

THEOREM 2.

$$
\sup _{B=B^{*} \in L(H)} \operatorname{dist}(\sigma(B), \operatorname{Re} \sigma(B+i A))=\frac{2}{\pi} \sum_{s=1}\left(\lambda_{s}^{+}+\lambda_{s}^{-}\right) /(2 s-1) .
$$

Proof. It follows from the Macaev theorem [3, Theorem III.4.2], or from Corollary 1 that "sup" is not less than the right-hand side. Thus it suffices to prove that if $B=B^{*} \in L(H)$ then

$$
\operatorname{dist}(\sigma(B), \operatorname{Re} \sigma(B+i A))<\frac{2}{\pi} \sum_{s=1}\left(\lambda_{s}^{+}+\lambda_{s}^{-}\right) /(2 s-1) .
$$

It follows from the Weyl-von Neumann theorem [6, Theorem X.2.1] that there exists a compact selfadjoint operator $K$ such that the operator $B+K$ has a pure point spectrum. Then there exists a sequence $\left\{P_{n}\right\}_{1}^{\infty}$ of orthogonal projections in $H$ converging strongly to the identity operator such that $\boldsymbol{P}_{\boldsymbol{n}}$ is $n$ dimensional and commutes with $B+K$. Define the operators $B_{n}, C_{n}$ by the formulas

$$
B_{n}=\left(1-P_{n}\right) B\left(1-P_{n}\right)+P_{n} B P_{n}, \quad C_{n}=B_{n}+i P_{n} A P_{n} .
$$

Since $B_{n}-B=P_{n}\left(K-K P_{n}\right)+\left(K-P_{n} K\right) P_{n}$ it follows from the compactness of $K$ that $\left\|B_{n}-B\right\| \rightarrow 0$. Since $A$ is compact too we see that $\left\|C_{n}-(B+i A)\right\| \rightarrow 0$. The operators $B_{n}, C_{n}$ are compact perturbations of $B$; therefore their essential spectra are identical. These facts and the perturbation theorems [6, Chapter IV, §3] imply that

$$
\operatorname{dist}\left(\sigma(B), \sigma\left(B_{n}\right)\right) \rightarrow 0, \quad \operatorname{dist}\left(\sigma(B+i A), \sigma\left(C_{n}\right)\right) \rightarrow 0 .
$$

Note further that $\sigma\left(B_{n}\right)=\sigma\left(\left.\left(1-P_{n}\right) B\right|_{\left(1-P_{n}\right) H}\right) \cup \sigma\left(\left.P_{n} B\right|_{P_{n} H}\right)$, and $\sigma\left(C_{n}\right)=$ $\sigma\left(\left.\left(1-P_{n}\right) B\right|_{\left(1-P_{n}\right) H}\right) \cup \sigma\left(\left.P_{n}(B+i A)\right|_{P_{n} H}\right)$. Consequently,

$$
\begin{aligned}
& \operatorname{dist}\left(\sigma\left(B_{n}\right),\right.\left.\operatorname{Re} \sigma\left(C_{n}\right)\right) \leqslant \operatorname{dist}\left(\sigma\left(\left.P_{n} B\right|_{P_{n} H}\right), \operatorname{Re} \sigma\left(\left.P_{n}(B+i A)\right|_{P_{n} H}\right)\right) \\
& \leqslant \frac{1}{n} \sum_{s=1}^{[n / 2]}\left(\lambda_{s}^{+}+\lambda_{s}^{-}\right) \operatorname{ctg} \frac{2 s-1}{2 n} \pi<\frac{2}{\pi} \sum_{s=1}^{\infty}\left(\lambda_{s}^{+}+\lambda_{s}^{-}\right) /(2 s-1),
\end{aligned}
$$

since the $j$ th positive (negative) eigenvalue of $\left.P_{n} A\right|_{P_{n} H}$, if it exists, is not greater (less) than $\lambda_{j}^{+}\left(-\lambda_{j}^{-}\right)$, and $\operatorname{ctg}((2 s-1) / 2 n) \pi<2 n /(2 s-1) \pi$. The desired inequality (7) now follows from (8) and (9).

\section{REFERENCES}

1. I. C. Gohberg, On connections between Hermitian components of nilpotent matrices and on an integral of triangular truncation, Bul. Akad. Śtiince RSS Moldoven. 1 (1963), 27-37. MR 35 \#2168. (Russian)

2. I. C. Gohberg and M. G. Krein, Introduction to the theory of linear nonselfadjoint operators in Hilbert space, "Nauka”, Moscow, 1965; English transl., Transl. Math. Monos., vol. 18, Amer. Math. Soc., Providence, R. I., 1969. MR 36 \#3137; MR 39 \# 7447. 
3. __ Theory of Volterra operators and its applications, "Nauka", Moscow, 1967; English transl., Transl. Math. Monos., vol. 24, Amer. Math. Soc., Providence, R. I., 1970. MR 36 \# 2007; MR 41 \#9041.

4. W. Kahan, Every $n \times n$ matrix $Z$ with real spectrum satisfies $\|Z-Z *\|<\|Z+Z *\|\left(\log _{2} n+\right.$ 0.038), Proc. Amer. Math. Soc. 39 (1973), 235-241. MR 47 \# 1833.

5. __ Spectra of nearly Hermitian matrices, Proc. Amer. Math. Soc. 48 (1975), 11-17. MR 51 \#5627.

6. T. Kato, Perturbation theory for linear operators, Die Grundlehren der math. Wissenschaften, Bd. 132, Springer-Verlag, New York, 1966. MR 34 \#3324.

Institute of Mathematics, Polish Academy of Sciences, 00-950, Warsaw, P. O. B. 137, Poland 